\title{
Design of electronic equipment fault diagnosis training system based on graphical platform
}

\author{
Zhiyong Cheng ${ }^{1, a}$, Zhongtao Zhu ${ }^{1, b}$, Rongyue Xie ${ }^{1, c}$, Deqiang Shu ${ }^{1, d}$, Qiu Lei ${ }^{1, e}$ \\ ${ }^{1}$ Wuhan Mechanical Technology College, Wuhan, 430075, China \\ aemail: zycheng72@sina.com, bemail: zhuzt@163.com, email: ryxie@126.com, \\ demail: Deqiangshu@sohu.com, eemail: hnnhlxy@163.com
}

Keywords: Fault diagnosis; Graphical; Platform; Layout algorithm; Orthogonal to the attachment.

\begin{abstract}
According to the characteristics of the electronic equipment fault diagnosis and maintenance training, graphical fault diagnosis system was designed. That the graphical training system of fault diagnosis composition, database design and data graphical representation was mainly introduced. The system adopted the design of platform, and it was versatile. Replaced the background data can realize different electronic equipment's fault diagnosis.
\end{abstract}

\section{Introduction}

With the development of electronic technology and the application of new technology, new electronic equipment has the following characteristics: (1) Electronic equipment update speedily. Electronic equipment replacement, large to the technical system of the equipment, composition structure disruptive change, small to the chip which used high performance, circuit, sensor or appearance and other local changes will result in electronic equipment upgrade, from civil electrical appliances, mobile phone to military weapons and equipment.(2)Higher requirements for the maintenance and support of electronic equipment. Due to the application of new technology and equipment is more and update speedily, so in order to maintain the good performance of the equipment during the service life, we need strong maintenance support , This puts a new task to the maintenance personnel's quality and the way of maintenance.

In this paper, we propose a graphical training system for the fault diagnosis of electronic equipment, and give a feasible solution to improve the maintenance personnel capability.

\section{The overall design}

In electronic equipment maintenance support, the common fault mechanism of typical fault, used in troubleshooting ideas, devices using instrument measuring tools and replace parts is usually fixed, to solve this type of failure, can greatly reduce the repair time, ensure the equipment quickly and then put into battle, while reducing the dependence on the higher maintenance departments, have important position in the first line of use departments. Therefore, it is very important to train the electronic equipments with common typical fault.

The fault maintenance training of electronic equipment, can adopt physical method to maintenance training. The method has the advantages of real training environment, obvious failure phenomenon, and direct maintenance effect. However, because of the narrow space of equipment, can only allow some personnel into the equipment one time, teaching is difficult, and due to the complexity of equipment, the maintenance content need to be trained repeatedly to master the essentials of maintenance, which is caused the low efficiency of training; second expensive equipment due to the repeated use operation and fault set during maintenance training cause its life shortens greatly, serious performance degradation. Therefore, it is urgent to need a economic and effective training methods to solve the teaching problem of the maintenance personnel.

Through the summary of fault maintenance training rules, the results show that, after observing the failure phenomenon, the idea of maintenance is is correct or not determines the success or failure of maintenance, so we design the interactive graphics of the fault tree training system to train 
its maintenance ideas.

The overall structure of the training system consists of the following two aspects. One is the graphical representation of the data (application part), and the second is the data store. For troubleshooting, in order to comply with the actual and easy to implement the electronic training, we adopt the fault tree diagram expression; In the mode of data storage, uses a database and multimedia way; For the fault tree, the failure phenomenon, failure detection and fault handling's exclusion process, through relational database to express storage (database), in order to maximize the close to the actual, for fault detection and processing using video, animation way to express (Multimedia), test results using static images show. The relationship between figure expression and data part is shown in Fig.1.

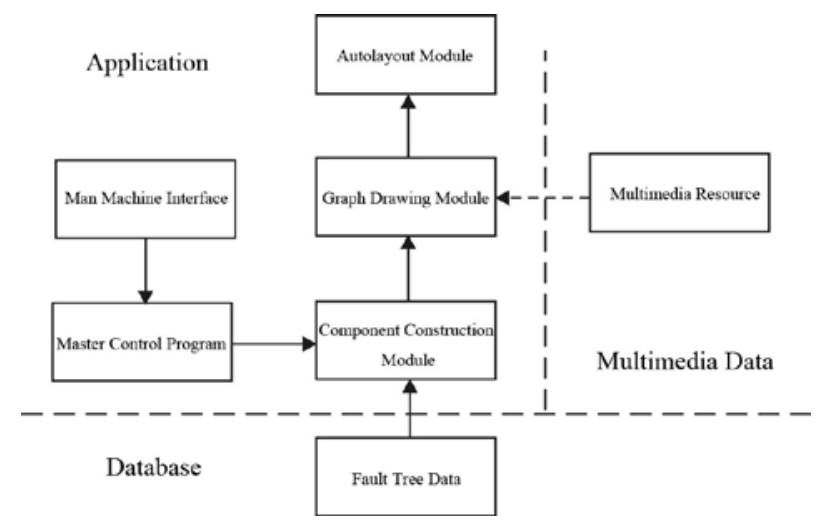

Fig.1. Graphics training system structure

Users through the man-machine interface to choose the fault need to be trained, the main control program will forward the user's choice to component building blocks, component building blocks read data in the database, after analysis, according to the rules to generate a series of detection components (corresponding to the fault tree detection) and processing components (corresponding to the fault tree processing), the component data through graphic drawing module to generate graphics components, the user interacts with the graphics components, can display multimedia resources which are related to graphics components.

\section{The implementation}

\section{Database design}

Database is an important part of graphical training system, the organization of data directly affects the graphical system efficiency and database maintenance complexity.

In view of the structure of the fault tree, the entity relationship diagram of fault, fault detection, test results and fault handling is abstracted, and the relationship is shown in Fig.2. One fault contains multiple fault detection, a detection items have to two kinds of test results, one kind of test results or connect one new detection item, or connect of one processing item.

In this fault tree database design, the table structure of the fault detection and detection results is the key and difficult point. The reason is that the two tables contain important information to generate the fault tree. Secondly, there is a relationship of inclusion and dependency between the two tables. The table structure of the fault detection is shown in table 1.

Table 1 table structure of fault detection

\begin{tabular}{|c|c|}
\hline Field & Instructions \\
\hline Test result code & (unique number) \\
\hline Test item code & $\begin{array}{c}\text { (test results corresponding to the } \\
\text { test items) }\end{array}$ \\
\hline Judge & (T and F) \\
\hline Resource information & (resource file path) \\
\hline
\end{tabular}




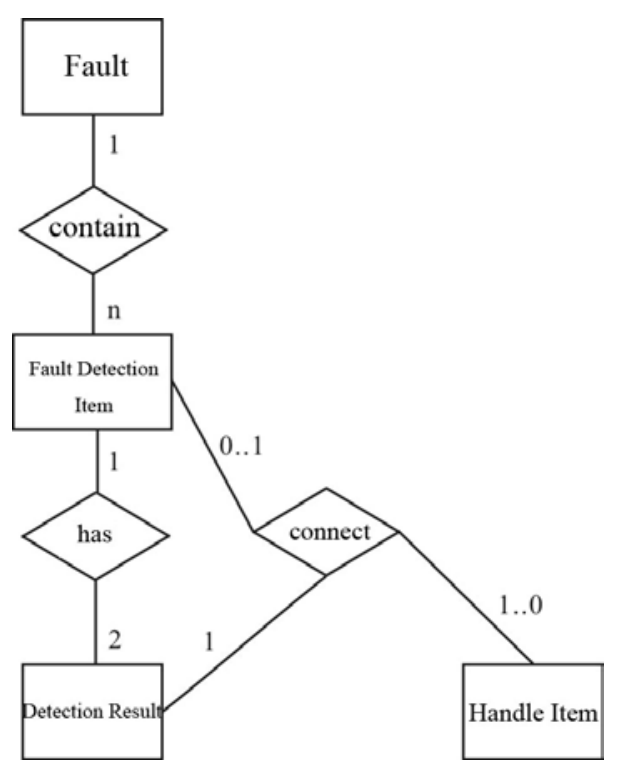

\section{The application design}

Fig.2. Entity relationship diagram

In the operation of the user, the application according to the selection of fault, through the database interface module to read and analyze the database data, and then through the component to build the module, the data are assembled into a testing component and processing components.

Graphics rendering module is mainly used to draw the test components and process components, when drawing uses rhombus to testing component, rectangular to processing components, and uses the orthogonal linear connection graphical component and express troubleshooting ideas.

Component drawing module in addition to display components in the window to build a module assembly of components, the other important function is to respond to user interaction, its response to user operation ways mainly have three kinds: 1 , double-click. The user can double-click the graphical component, it can display the graphics component associated with the multimedia information (detection, detection results or processing of the video, pictures), and its multimedia information display as shown in figure 3; Method 2, drag and drop. Users drag and drop the component in the application, components can be achieved by the location of the mobile; 3 , the connection. The user through the analysis of the results of the test, to connect another test item or a certain processing, to achieve fault analysis and processing of thinking training, and troubleshooting training attachment as shown in figure 4.

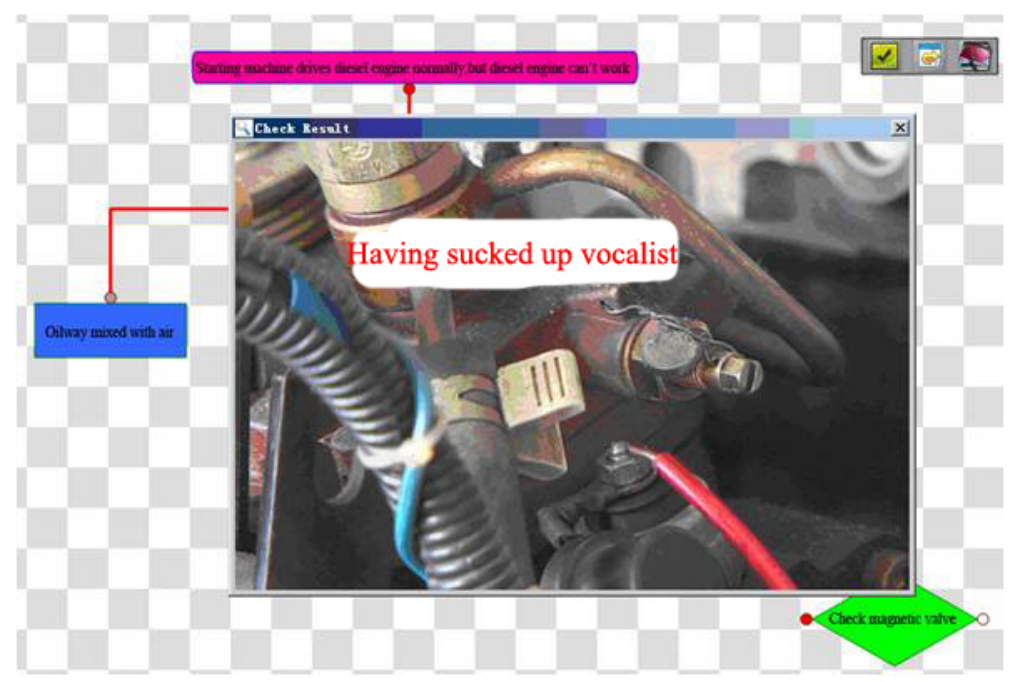

Fig.3. Multimedia resource displays 


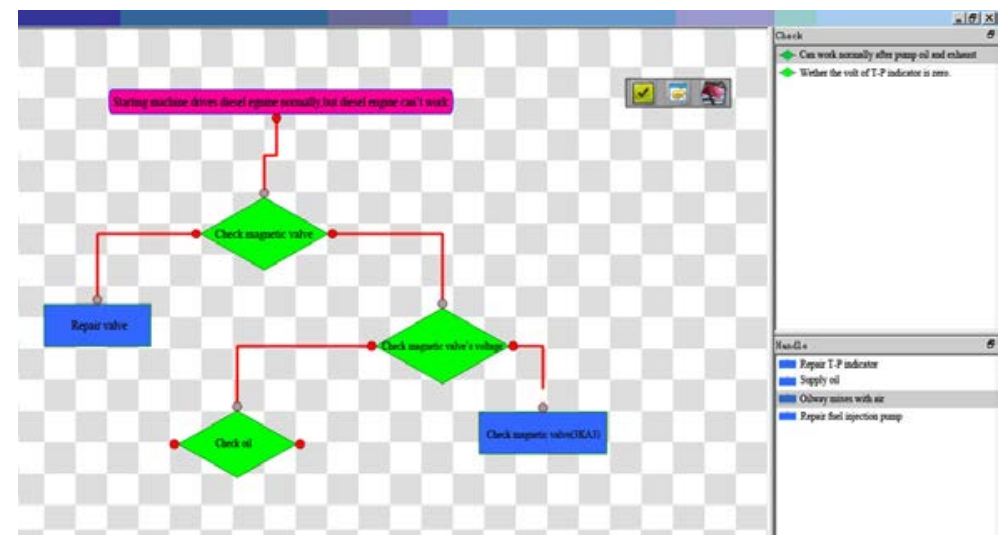

Fig.4. Fault diagnosis training

At the same time the training module provides an optimized fault tree based on summarize the actual maintenance, can be used as reference for the user training, the one of the difficult points of the software design is to make the graphics of different fault tree reasonable displayed in the application window graphic layout module, graphic layout has the following constraints: 1 . Graphical components can't be overlapped; 2. Connection line between minimize perfunctory; 3. The fault tree has a basic level of alignment. Based on the above constraints, the tree layout algorithm is selected, which can be used in good effect and high efficiency. The automatic layout of the algorithm is shown in Fig.5.

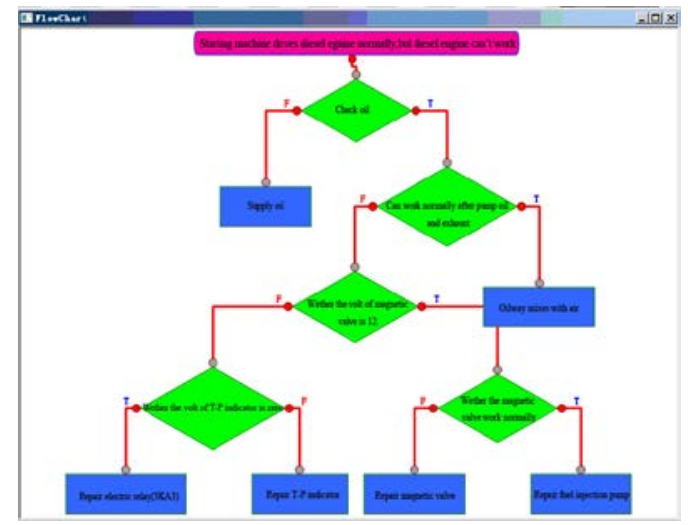

Fig.5. Automatic layout effect diagrams

\section{Conclusion}

This training system database used SQLite database management system, the application program was developed under the Qt framework by $\mathrm{C}++$ language. The system had friendly man-machine interface, easy to operate, and the information was direct. The system used the modular design, the application design became the independent platform, the user only needed to add, modify the database data, and then could realize the graphics training of the corresponding electronic equipment's fault diagnosis.

\section{References}

[1] Lan Jialong, Liu Jun. Application of graph theory and algorithm. [M] Chengdu: Electronic Science and Technology University Press, 1995:72 132

[2] Cai Hengwen, Yan Zhenglong, Zhang Li. Principle and application of database system [M] Nanchang: Jiangxi University Press, 2004:17 74

[3] Yin Renkun. Data structure: using object-oriented method and C++ language description [M] (second edition). Beijing: Tsinghua University press, 2007:297-396.

[4] Yu Dahai, Liu hao. The design and implementation of interactive electronic manual based on the 
failure mode. [J] Journal of Sichuan ordnance, 2010, Volume 31:28 30.

[5] Zhao Guoqing, Yang Nanying, Jia Zhenyang et al. Research on the concept map's layout algorithm. [J] Open education research, 2005, 11 (5): $32 \sim 37$.

[6] Jasmin Blanchette,Mark Summerfiled. C++ GUI Programming with Qt4, Second Edition. Beijing: Electronic Industry Press, 2007:129 182 